\title{
Devenir dominant
}

Les grandes étapes de l'expérience de la mobilité sociale ascendante

Jules Naudet

\section{CpenEdition}

Journals

Édition électronique

URL : http://journals.openedition.org/ress/1182

DOI : $10.4000 /$ ress. 1182

ISSN : 1663-4446

Éditeur

Librairie Droz

\section{Édition imprimée}

Date de publication : 15 juin 2012

Pagination : 161-189

ISBN : 978-2-600-01613-1

ISSN : 0048-8046

Référence électronique

Jules Naudet, "Devenir dominant », Revue européenne des sciences sociales [En ligne], 50-1 | 2012, mis en ligne le 15 juin 2015, consulté le 23 avril 2019. URL : http://journals.openedition.org/ress/1182 ;

DOI : 10.4000/ress. 1182 


\title{
DEVENIR DOMINANT LES GRANDES ÉTAPES DE L'EXPÉRIENCE DE LA MOBILITÉ SOCIALE ASCENDANTE
}

\author{
JULES NAUDET \\ ERIS, CMH (CNRS-EHESS-ENS) \\ julesnaudet@hotmail.com
}

\begin{abstract}
Résumé. Cet article s'appuie sur une enquête menée auprès d'une centaine de personnes originaires de milieu populaire et ayant connu une très forte ascension sociale en France et aux États-Unis. II propose une typologie des principales étapes de l'expérience de la mobilité sociale, prenant appui sur l'analyse phénoménologique de l'étranger développée par Alfred Schütz. Cette typologie permet de saisir à quel point l'expérience de la tension entre milieu d'origine et milieu d'arrivée varie fortement en fonction des étapes de la trajectoire d'ascension. Cette absence d'un effet unique de la mobilité doit ainsi amener à un renouvellement partiel des paradigmes structurant la recherche sur l'expérience de la mobilité sociale: en fonction de l'âge des personnes étudiées, les effets que font ressortir les travaux peuvent varier considérablement. La période autour des études supérieures condense notamment toutes les contradictions auxquelles doivent faire face les personnes en ascension sociale.
\end{abstract}

Mots-clés: mobilité sociale ascendante, expérience, âges de la vie, trajectoire sociale.

\begin{abstract}
This article is based on a survey conducted among a hundred persons from a working-class background who achieved very sharp upward social mobility. It proposes a typology of the main stages in the experience of social achievement, drawing on the phenomenological analysis of the stranger developed by Alfred Schütz. This typology shows that the experience of the tension between the group of origin and the new group varies strongly depending on the stages of the mobility trajectory. The fact that there is no single effect of mobility must lead to a partial renewal of the paradigms structuring the research on the experience of social mobility. Indeed, depending on the age of the people surveyed, conclusions can vary widely. The disruptive effects of upward mobility are felt most acutely when going through higher education.
\end{abstract}

Keywords: upward social mobility, experience, life stages, social trajectory. 
Les débats sur les «conséquences de la mobilité sociale» sont généralement structurés autour de deux pôles théoriques: l'hypothèse de «dissociation» et l'hypothèse d'«acculturation» (Naudet, 20I I). La première, qui trouve ses racines dans l'idée durkheimienne de l'anomie comme conséquence d'un changement social rapide ainsi que dans les réflexions de Pitirim Sorokin', pose que la forte mobilité sociale entraîne des troubles identitaires et mentaux. La seconde, dont le représentant principal est Peter Blau², insiste davantage sur les changements d'attitudes et de comportement associés à l'expérience de la mobilité, à travers une identification au groupe d'arrivée, que sur les coûts subjectifs qui lui sont associés. L’hypothèse d’acculturation, soit l'idée que le succès est tellement valorisé dans la société que les personnes en mobilité cherchent davantage à se conformer à leur groupe d’arrivée qu'à rester fidèles à leur groupe d'origine, domine largement les études quantitatives menées aujourd'hui. À l'opposé, les études dites «qualitatives» se trouvent être, dans l'ensemble, beaucoup plus proches du paradigme durkheimien ${ }^{3}$. Si la sociologie quantitative insiste sur les processus d'acculturation ou sur des attitudes de «surconformité» [overconformity], la sociologie «qualitative» insiste donc davantage sur les risques de «clivage du moi», de «névrose de classe», de «dédoublement», etc. auxquels sont exposées les personnes en ascension sociale.

Cependant, d'un côté comme de l'autre, la croyance en un effet unique de l'ascension sociale est entretenue. Or, en voulant saisir une unique «conséquence de la mobilité», qu’il s’agisse de ses coûts ou de ses bénéfices, on s'empêche de saisir en quoi l'expérience de la mobilité varie au cours de la trajectoire. Une sociologie compréhensive de l'expérience de la mobilité sociale doit en effet tenir compte

I Sorokin (1927, p. 515) affirme ainsi: "Great mental strain and versatility of behavior, demanded by life in a mobile society, are so exacting that they can not be met by many individuals. Their nervous systems crumble under the burden of the great strains required of them. Hence arises the increase of mental disease and nervousness, psychoses and neurosis".

2 Selon Blau (1956, p. 293) "the upwardly mobiles differ widely [...] from members of their class of origin (the lows) and seem to overconform with the practices prevalent among their new social class (the highs)". Voir également Blau et Duncan (1967). Pour Frederick Turner (1992, p. 192), qui défend la même idée, "The ties between attitudes and the past experience of mobility at the individual level are more apparent than real". Nous renvoyons également à Luckmann et Berger (1964).

3 Pour une revue de littérature détaillée sur ce sujet, voir Naudet, 201I. 
d'éventuels effets d'âge. Cette proposition est notamment renforcée par le fait que les travaux qui offrent les plaidoyers les plus solides en faveur de la perspective durkheimienne s'appuient souvent sur des échantillons constitués d'étudiants ou de personnes en début de carrière. Ainsi, pour ne prendre que ces exemples, lorsque Richard Hoggart (1970) met en évidence les difficultés auxquelles est confronté le «boursier», il décrit des personnes qui sont encore étudiantes ou tout récemment entrées dans le monde du travail ${ }^{4}$. De même, les travaux de Robert Ellis et Clayton Lane (1967) ou d'Earl Hopper (198I) soutiennent l'hypothèse de dissociation et d'anomie à partir d'études quantitatives menées sur des populations étudiantes. Ces simples remarques nous amènent à poser l'hypothèse, peu évoquée dans les débats théoriques, selon laquelle les conséquences négatives de l’ascension sociale seraient plus intenses à certaines étapes de la trajectoire qu'à d’autres.

Nous nous situons donc dans le prolongement de travaux qui distinguent plusieurs phases dans la trajectoire de mobilité ascendante. C'est notamment le cas de l'ouvrage d'Alfred Lubrano (2004), qui s'appuie sur une centaine d'entretiens auprès de «cols bleus» devenus «cols blancs», et dont chacun des chapitres présente un moment précis de la trajectoire de mobilité. Richard Zweigenhaft et William Domhoff (2003) s'efforcent également d’identifier l'évolution identitaire des personnes qui sont issues du programme $\mathrm{ABC}$ ( $\mathrm{A}$ better chance) et distinguent trois périodes marquées par des expériences singulières: la «prep school», l’université et la carrière professionnelle. On retrouve cette même idée d'étapes chez Jean-Pierre Terrail (1990), qui construit son analyse de l'expérience de la mobilité sociale autour des principales périodes de la trajectoire scolaire: le primaire, le collège, le lycée, l’université et l'après-université. De manière générale, tous les travaux qui s'efforcent

4 Dans son «portrait de Richard Hoggart en sociologue», Jean-Claude Passeron (1993) résume ainsi les types d'intellectuels issus des classes populaires que distingue Richard Hoggart dans son œuvre: il y aurait les «boursiers» (« décidés à la réussite ou résignés à la demi-réussite»), les «ratés pathétiques» («voués à la névrose d'échec vécue comme une guigne») et enfin les «parvenus» («qui "en remettent" un rien de trop dans une virtuosité où se trahit l'emprunt, hommes habités par le ressentiment ressassé ou la capitulation amère, lettrés ou savants installés dans l'establishment au prix d'un oubli de leur origine, assez proches finalement, par la véhémence de leur dénégation silencieuse, des self-made-men ou des diplômés hâbleurs qui cultivent l'autoglorification par la magnification des vertus intellectuelles et morales prêtée à une ascension miraculeuse opérée à partir du néant social»). 
de retracer des biographies de personnes en mobilité sociale ascendante, ainsi que toutes les autobiographies de personnes en mobilité sociale, mettent en évidence différentes étapes dans l'expérience de la mobilité sociale, même s’ils ne cherchent pas explicitement à les distinguer conceptuellement au travers de types idéaux.

Ces travaux demeurant essentiellement descriptifs, et n'interrogeant pas suffisamment l'évolution du rapport entretenu au milieu d'origine, nous entendons donc proposer une nouvelle typologie des grandes étapes de l'expérience de la mobilité sociale. Notre contribution prend comme point de départ l'analyse phénoménologique de la situation de l'«étranger» par Alfred Schütz (2003). De la même manière qu'il détermine différentes phases dans la trajectoire idéaltypique de l'«étranger», il est possible d'identifier plusieurs étapes de l'expérience de la tension entre milieu d'origine et milieu d’arrivée au cours de la trajectoire de mobilité. Nous défendons plus précisément l’idée que ces «grandes étapes de l'expérience de la mobilité sociale» peuvent être identifiées dans des contextes sociaux et culturels aussi variés que ceux des États-Unis et de la France. Après une présentation du matériau sur lequel nous nous appuyons et un court retour sur l'article d'Alfred Schütz, nous proposerons une typologie des cinq principales étapes de l'expérience de la mobilité sociale ascendante.

\section{L'ENQUÊTE}

Nous nous appuyons ici sur 5I entretiens réalisés en France (en 2004 puis en 2008-2009) et 42 entretiens aux États-Unis (entre avril 2007 et septembre 2007) auprès de hauts fonctionnaires, de personnes occupant des positions élevées dans le secteur privé et d’universitaires 5 . Les parents des interviewés français et américains étaient tous ouvriers ou employés peu qualifiés. Les parents des interviewés indiens étaient agriculteurs sans terre, petits paysans, ouvriers, travailleurs manuels ou employés peu qualifiés.

5 L'enquête initiale de laquelle est tiré cet article comprenait également 57 entretiens réalisés en Inde (Naudet, 20 I0). Si l'analyse des entretiens menés en Inde confirme les analyses présentées ici, nous ne nous appuyons pas dessus dans cet article. La présentation des travaux menés en Inde aurait en effet nécessité une mise en contexte que le format de cet article ne nous permettait pas de réaliser. 
Aux États-Unis (voir tableau I), les hauts fonctionnaires sont tous membres du SES (Senior Executive Service), soit l'un des plus hauts rangs qu'il est possible d'atteindre dans le service public fédéral. Les personnes travaillant dans le secteur privé occupent toutes des positions très élevées et ont un diplôme de niveau master ou un MBA. Les universitaires ont tous un doctorat en sciences humaines ou en sciences sociales et sont enseignants-chercheurs.

En France (voir tableau 2), les hauts fonctionnaires sont tous des anciens élèves de l'ENA (École Nationale d'Administration). Les personnes travaillant dans le secteur privé sont toutes issues de Grandes écoles d'ingénieur et de commerce ou sont d'anciens énarques. Les universitaires sont des chercheurs ou des enseignants-chercheurs titulaires d'un doctorat en sciences sociales ou en sciences humaines. Ces entretiens sont complétés par io autres, réalisés en 2003, auprès d'enfants d'ouvriers étudiants à Sciences-Po Paris (voir tableau 3).

Tableau I : Entretiens réalisés aux États-Unis entre avril 2007 et septembre $2007^{6}$

\begin{tabular}{|c|c|}
\hline & ÉTATS-UNIS \\
\hline \multirow{2}{*}{ UNIVERSITÉ/RECHERCHE } & 16 \\
\cline { 2 - 2 } & dont african-americans: 5 \\
\hline \multirow{2}{*}{ HAUTE FONCTION PUBLIQUE } & 16 \\
\cline { 2 - 2 } & dont african-americans: 2 \\
\hline \multirow{2}{*}{ SECTEUR PRIVÉ } & 10 \\
\cline { 2 - 2 } & dont african-americans: 5 \\
\hline TOTAL INTERVIEWS & 42 \\
\cline { 2 - 2 } & dont african-americans: 12 \\
\hline
\end{tabular}

6 Toutes classes sociales confondues, il n'y avait que 7\% d'Afro-américains à des positions SES en 1995 (Naff, 1998). Sur les Afro-américains dans les Executive Branch Agencies, voir aussi Page, 1994. 
Tableau 2: Entretiens réalisés en France

\begin{tabular}{|c|c|c|c|}
\hline & $\begin{array}{l}\text { FRANCE (ENTRETIENS RÉALI- } \\
\text { SÉS EN 2008-2009) }\end{array}$ & $\begin{array}{l}\text { FRANCE (ENTRETIENS } \\
\text { RÉALISÉS EN 2004) }\end{array}$ & TOTAL \\
\hline \multirow[b]{2}{*}{$\begin{array}{l}\text { UNIVERSITÉ/ } \\
\text { RECHERCHE }\end{array}$} & 8 & 8 & 16 \\
\hline & $\begin{array}{l}\text { Dont personnes originaires } \\
\text { d'anciennes colonies } \\
\text { françaises: } 0\end{array}$ & $\begin{array}{l}\text { Dont personnes originaires } \\
\text { d'anciennes colonies } \\
\text { françaises: } 3\end{array}$ & $\begin{array}{l}\text { Dont personnes originaires } \\
\text { d'anciennes colonies } \\
\text { françaises: } 3\end{array}$ \\
\hline \multirow[b]{2}{*}{$\begin{array}{l}\text { HAUTE } \\
\text { FONCTION } \\
\text { PUBLIQUE }\end{array}$} & 18 & 0 & 18 \\
\hline & $\begin{array}{l}\text { Dont personnes originaires } \\
\text { d'anciennes colonies } \\
\text { françaises: I }\end{array}$ & $\begin{array}{l}\text { Dont personnes originaires } \\
\text { d'anciennes colonies } \\
\text { françaises: } 0\end{array}$ & $\begin{array}{l}\text { Dont personnes originaires } \\
\text { d'anciennes colonies } \\
\text { françaises: I }\end{array}$ \\
\hline \multirow{3}{*}{$\begin{array}{l}\text { SECTEUR } \\
\text { PRIVÉ }\end{array}$} & 10 & 7 & 17 \\
\hline & $\begin{array}{l}\text { Dont personnes originaires } \\
\text { d'anciennes colonies } \\
\text { françaises: } 0\end{array}$ & $\begin{array}{l}\text { Dont personnes originaires } \\
\text { d'anciennes colonies } \\
\text { françaises: I }\end{array}$ & \multirow{2}{*}{$\begin{array}{l}\text { Dont personnes originaires } \\
\text { d'anciennes colonies } \\
\text { françaises: I }\end{array}$} \\
\hline & $\begin{array}{l}\text { Dont anciens élèves de } \\
\text { I'ENA: } 6\end{array}$ & $\begin{array}{l}\text { Dont personnes originaires } \\
\text { du Portugal: } 4\end{array}$ & \\
\hline \multirow[b]{2}{*}{$\begin{array}{c}\text { TOTAL } \\
\text { INTERVIEWS }\end{array}$} & \multirow[b]{2}{*}{36} & \multirow[b]{2}{*}{15} & 51 \\
\hline & & & $\begin{array}{l}\text { Dont personnes originaires } \\
\text { d'anciennes colonies } \\
\text { françaises: } 5\end{array}$ \\
\hline
\end{tabular}

Tableau 3: Autres sources utilisées pour le terrain français

\begin{tabular}{|l|c|}
\hline \multicolumn{1}{|c|}{ TYPE DE SOURCE } & \multicolumn{1}{c|}{ EFFECTIFS } \\
\hline Questionnaires auprès d'anciens élèves de l'ÉNA & 31 \\
\hline $\begin{array}{l}\text { Entretiens auprès d'étudiants de Sciences-Po Paris issus } \\
\text { de milieu ouvrier (enquête 2002-2003) }\end{array}$ & $\begin{array}{l}\text { Dont quatre personnes originaires } \\
\text { d'anciennes colonies françaises et une } \\
\text { personne originaire de Turquie. }\end{array}$ \\
\cline { 2 - 2 }
\end{tabular}


La majorité des interviewés ont été contactés à la suite de la publication d'annonces sur des réseaux d’anciens élèves ou sur des lettres de diffusion de réseaux professionnels. Nous avons également eu recours à la technique du snowball sampling pour compléter l'échantillon des sous-groupes pour lesquels le nombre de réponses aux annonces était insuffisant.

Les entretiens réalisés s'appuyaient sur un guide d'entretien biographique, s'achevant par des questions plus réflexives. Ce guide d'entretien était identique dans les deux pays. Les interviewés étaient prévenus que l'enquête portait sur leur trajectoire de forte mobilité ascendante, et cela influençait nécessairement l'orientation générale de l'entretien. Celui-ci débutant par des questions sur l'enfance en milieu populaire, la connaissance préalable de l'objet de notre recherche avait pour conséquence de pousser l'interviewé à expliciter comment, à partir de la situation évoquée en début d'entretien, il a atteint son statut professionnel actuel. Dès lors, le matériau recueilli est particulièrement propice à l'identification de régularités dans la façon dont les personnes en mobilité choisissent de mettre en récit leur trajectoire de réussite. De plus, comme le dénouement du récit est forcément heureux, ou plutôt jamais complètement malheureux (il y a une «réussite sociale» à la clé), les interviewés s'autorisaient souvent à émailler leur narration de l'évocation de moments difficiles, de périodes de crise ou de doute, ou d'autres éléments moins glorieux ${ }^{7}$.

\section{LE CADRE THÉORIQUE : ALFRED SCHÜTZ ET L'ÉTRANGER}

Pour analyser les récits de l'expérience de la mobilité sociale de nos interviewés, nous nous appuyons sur le cadre théorique proposé par Alfred Schütz (2003) dans son analyse idéal-typique de la position de «l'étranger». Celle-ci invite en effet à développer une conception «dynamique » de la façon dont se déroule le processus de déculturation et d'acculturation auquel est soumise la personne en forte ascension sociale.

7 À l'inverse il est beaucoup plus difficile de réaliser des entretiens avec des personnes en mobilité descendante (Newman, 1989; Peugny, 2009). 
Schütz explique ainsi que, dans un premier temps, l'étranger aborde généralement le nouveau groupe à partir du schéma interprétatif de son groupe d'origine. Avant d'entrer dans le nouveau groupe, son attitude est celle d'un spectateur désintéressé. L’arrivée dans le nouvel environnement marque une première rupture en ce qu'elle implique le passage de la posture de spectateur à celle de membre potentiel : «Bondissant, pour ainsi dire, de la salle sur la scène, l'ancien spectateur devient un membre de la troupe » (Schütz 2003, p. 22).

Dans un second temps, le nouveau groupe devient moins lointain et les typologies toutes faites de l'étranger s'écroulent. Les représentations des objets étrangers à partir du schéma interprétatif du groupe d'origine ne coïncident plus avec l'expérience qui est faite de ces mêmes objets sociaux dans leur environnement.

Dans un troisième temps, la représentation toute faite du nouveau groupe qui existe au sein du groupe d'origine se révèle tout simplement être inadéquate. S’apercevant que les représentations de son groupe servent «uniquement de schéma d'interprétation commode du nouveau groupe et non de guide pour l'interaction entre les deux groupes » (ibid, p. 23), que dans ce cadre les membres du nouveau groupe ne sont que des objets et non pas des sujets, le nouveau venu voit sa confiance dans sa «façon de penser habituelle » ébranlée. L'étranger doit donc avoir recours à une «traduction» des schémas d'interprétation et d'expression du nouveau groupe dans les termes de son propre modèle culturel.

Ce n'est qu'après avoir saisi et compris «les subtilités interprétatives du nouveau modèle culturel que l'étranger peut commencer à l'adopter comme schéma de son expression personnelle» (ibid., p. 27). La description qu'il fait de cette dernière phase de familiarisation avec le nouveau groupe est particulièrement riche. Schütz laisse entendre que le rapport de l'étranger à son nouveau modèle culturel est toujours différent du rapport que les membres internes entretiennent vis-à-vis de leur groupe: «Hésitant et perplexe, il se méfie de tout ce qui apparaît d'une simplicité élémentaire à ceux qui se fient à l'efficacité des recettes qu'il suffit de suivre, sans se poser de questions, et sans comprendre. » (ibid., p. 35). Pour Schütz, l'étranger est constamment dans une position d'enquêteur vis-à-vis du nouveau groupe, ce qui explique que l'attitude de l'étranger envers le groupe est marquée par deux traits principaux: 
une objectivité de l'étranger; et une loyauté ambiguë. Cette loyauté ambigüe découle notamment du fait que bien souvent l'étranger ne considère pas le nouveau groupe «comme un asile protecteur, mais bien plutôt comme un labyrinthe dans lequel il a perdu tout sens de l'orientation. » (ibid., p. 38).

À la fin de son article, Alfred Schütz laisse néanmoins ouverte la possibilité d'une dernière étape, celle où l'étranger n'est plus étranger : si le processus d'enquête au sein du modèle culturel du nouveau groupe réussit, «alors ce modèle et ces éléments principaux deviendront pour le nouveau venu un simple état de fait, une manière de vivre allant de soi, un asile et une protection » (ibid., p. 39).

Nous entendons nous inspirer de l'analyse que propose Schütz dans notre étude des différents moments de l'expérience de la tension, tout en ajoutant au cadre d'analyse l'idée que ces étapes correspondent la plupart du temps à des âges de la vie bien précis.

\section{LES GRANDES ÉTAPES DE L'EXPÉRIENCE DE LA TENSION ENTRE MILIEU D'ORIGINE ET MILIEU D'ARRIVÉE}

\section{I.PREMIÈRE ÉTAPE: L'IDENTIFICATION AUX NORMES DE LA RÉUSSITE}

Avant que ne débute le processus d'acculturation au nouveau groupe, avant même toute socialisation anticipatrice au nouveau groupe, la mobilité sociale ascendante nécessite une première identification aux normes de la réussite. Il faut que l'enfant ressente une motivation suffisante pour travailler et réussir scolairement afin d’assurer ses succès futurs ${ }^{8}$. Cette partie demeure sans aucun doute la plus «mystérieuse» des étapes de l'expérience de la mobilité sociale,

8 Le cas des personnes ayant réussi sans l'aide de titres scolaires (que l'on nomme parfois «selfmade men»), nécessite un cadre d'analyse différent en ce que l'identification aux normes de réussite n'est pas forcément congruente avec l'identification aux normes scolaires. Pour le «selfmade man», il n'y a de succès que social ou économique, beaucoup plus rarement de succès scolaire. Notre étude portant sur des personnes dont la réussite est avant tout due à des titres scolaires, l'analyse que nous proposons ici se limite donc aux cas de ces personnes. L'article «Social Mobility: A Test of the Marginality and Acculturation Hypotheses» de Sheena Ashford (1989) pointe une différence nette entre l'expérience de la mobilité sociale liée à une réussite scolaire et l'expérience de la mobilité impliquant d'autres voies. Les travaux de Poliak (1992) sur les autodidactes laissent également deviner une expérience singulière des trajectoires de ces personnes. 
en ce qu'elle pose la question des déterminants de la réussite. Le chantier d'une «sociologie des irrégularités sociales» (Mercklé, 2005) reste ouvert et les sociologues n'ont pas fini de chercher comment rendre compte des «miraculés scolaires ». Qu'est-ce qui fait que certains ne calquent pas leurs dispositions subjectives sur leurs probabilités objectives de réussir (Bourdieu, 1974)? Pourquoi certains parviennent-ils à échapper aux «lois» de la reproduction sociale ? Ce chantier de recherche des «déterminants de la réussite » a conduit à de nombreuses études mais reste encore largement ouvert.

Cependant, l'identification des déterminants de la réussite scolaire et sociale ne suffirait pas à isoler ce qui, au sein de la socialisation primaire, permet de comprendre le rapport qui est entretenu plus tard au nouveau statut social ni, surtout, comment se réalisent les premières identifications aux normes de la réussite. Il est donc important de faire la distinction entre une sociologie de l'expérience de la réussite et une sociologie des déterminants de la réussite. C'est ainsi davantage dans les travaux de sociologues comme Annette Lareau (2000, 2003) aux États-Unis ou Bernard Lahire (1995) en France, dont les analyses cherchent avant tout à tracer les liens qui existent entre mode de socialisation et rapport à l'école, que l'on trouvera des pistes pour comprendre les enjeux qui se mettent en place dans cette première étape du parcours de mobilité.

La façon qu'ont nos interviewés d'expliquer comment s'est déroulée leur identification aux normes de la réussite scolaire et sociale a fait l'objet d'un article qui aborde cette question (Naudet, 2012) et nous ne développons donc pas davantage ce point ici. Il convient néanmoins de préciser que dans la narration qui est faite du parcours de réussite, cette étape est généralement propice aux reconstructions ex-post de l'histoire personnelle. La théorie de la psychanalyse de Freud (1973) dont s'inspire Vincent de Gaulejac (1987) met ainsi en avant l'importance de la réinvention des origines pour motiver le besoin de réussir. 


\section{2. DEUXIÈME ÉTAPE: DÉCOUVERTE DE L'ALTÉRITÉ SOCIALE ET PRISE DE CONSCIENCE DE L'ÉLOIGNEMENT PROGRESSIF DU MILIEU D'ORIGINE}

La deuxième étape que nous distinguons consiste en la prise de conscience de l'éloignement du milieu d’origine. Cette découverte de l'altérité sociale se réalise de manière progressive, notamment au travers de pratiques qui apparaissent de plus en plus dissonantes avec le milieu d'origine. Mais cette prise de conscience va aussi passer par des épisodes plus brutaux et «datables » comme lors de rencontres avec des personnes issues de groupes dominants.

L'école est souvent le lieu au sein duquel va se jouer cette lente découverte. Ainsi lorsque nous demandons à Mathias si ses parents l'aidaient pour son travail à la maison pendant sa scolarité primaire, il nous répond :

Non, pas du tout. En général quand j’avais des devoirs c'était: «Ouais, joue, profite, tu joueras plus quand t'auras quinze ans! Tu traîneras avec les filles...» Tu vois bien le genre d'arguments qui peuvent avoir cours... C'était pas non plus «bâcle tes devoirs!», mais c'était «ouais si tu les fais pas cette fois-ci, tant pis! C’est pas la mort!» (Mathias, étudiant à Sciences-Po, $4^{\mathrm{e}}$ année).

Cet exemple concentre toutes les contradictions qui peuvent exister entre les attentes de la famille de Mathias et les attentes de l'institution scolaire. L'accumulation de ce type d'expériences va progressivement amener le «bon élève » issu de milieu populaire à réaliser qu'il est en train de s'éloigner de sa famille. En effet, ce qui va de soi dans l'univers scolaire n’est pas nécessairement évident pour les personnes issues de milieux étrangers au monde du savoir. Au sein du nouveau schéma de référence que découvrent ces personnes, la vision importée du groupe natal s'avère souvent inadéquate ${ }^{9}$.

9 Un certain nombre de nos interviewés sont issus de familles qui, bien que «populaires», pouvaient valoriser fortement le savoir. Du fait de cette valorisation, ils ont grandi dans un environnement où les contradictions qui peuvent exister entre la famille et l'école apparaissent de manière moins évidente. De plus, la dimension «banale», quotidienne et «typique» du fait d'aller à l'école peut rendre plus difficile la prise de conscience du fait que la réussite scolaire implique, en partie, une prise de distance avec son milieu. 
La prise de conscience de l'éloignement du milieu d'origine est parfois moins progressive et plus brutale, et certaines confrontations avec des milieux sociaux différents jouent alors le rôle de révélateur de cet éloignement. De telles expériences de l'altérité sociale peuvent être vécues à des âges très différents en fonction des stratégies scolaires et résidentielles des parents. Cette prise de conscience brutale des hiérarchies sociales implique généralement une expérience de l'infériorité sociale, voire dans certains cas de l'humiliation. Deux éléments sont particulièrement importants pour comprendre cette première découverte de l’altérité sociale : le moment auquel elle est faite, et son intensité. En effet, celle-ci peut se faire de manière précoce, dès l'école primaire, ou de manière tardive, au cours du cursus d'études supérieures; et elle peut être plus ou moins brutale, ou plus ou moins progressive.

Pour Michael, cette découverte s'est faite au moment de son arrivée en Junior High School. Pour des raisons de stratégie scolaire, ses parents ont décidé de l'inscrire dans une Junior High School privée alors qu’il avait passé toute sa scolaritéprimaire dans une école dont la population était majoritairement ouvrière et constituée pour un tiers d'Afro-américains, pour un tiers d'Hispaniques et pour un tiers de Blancs. Il nous raconte comment son arrivée dans cette école a été immédiatement marquée par une prise de conscience brutale de son appartenance de classe :

Lors de ma première semaine dans cette école privée, je me rappelle que quelqu'un m’a dit: «Tu as déjà porté cette chemise mardi : tu n’as pas plus de chemises que ça?» Personne ne m’avait jamais dit ça de ma vie. Personne ne maavait jamais dit: «Michael, tu n’as que cinq chemises!! » Donc cela a été très difficile d'arriver dans un endroit vraiment différent en termes de classe. [...] C'était très dur pour moi la première année. Je n'avais pas beaucoup d'amis, je n'arrivais pas à m’adapter [I didn't fit in], j'étais dépendant des bourses : j’avais une bourse mais le deal c'était qu'en échange de cette aide financière je devais faire un certain nombre de choses pour le bureau du principal [Michael était le «mouchard», l'indicateur du principal]. Et je prenais le bus qui allait dans les quartiers pauvres, donc c'était assez évident de savoir qui j’étais. [...] Je n'invitais jamais personne chez moi parce que j'étais trop embarrassé par ma maison (Michael, 37 ans, doctorat de science politique, associate-professor). 
Si l'absence de conscience des différences sociales reste exceptionnelle, les personnes en mobilité peuvent néanmoins développer un certain nombre de stratégies pour minimiser l'impact de cette découverte. C'est notamment le cas des étudiants qui vont chercher à se regrouper entre semblables pour éviter de se retrouver dans des situations qui les renvoient trop violemment à leur «différence» sociale. Par ailleurs, cette découverte de l'altérité sociale est très fortement liée aux «voies de mobilités» qui sont empruntées. Il faut ainsi rappeler l'importance des stratégies résidentielles ou scolaires des parents: l'expérience des différences de classe est, en effet, fortement liée au type de quartier dans lequel on réside ou au type d'établissement dans lequel on étudie.

La découverte de l'altérité sociale s’accompagne généralement d’une prise de conscience de l'éloignement du milieu d'origine. En effet, en vivant une expérience qui n’a pas été vécue par ses parents et pour laquelle elle n’a pas été préparée, la personne en mobilité sociale prend conscience du fait qu'elle s'est engagée dans une voie qui n'est pas celle de ses parents et qui la mène quelque part où ses parents ne sont jamais allés. Néanmoins, cette première prise de conscience se fait encore du point de vue du milieu d'origine qui demeure le principal pôle de référence, et ce n'est que dans un temps ultérieur que le point de vue du milieu d’origine sera mis en question. La prégnance du point de vue du milieu d'origine ressort nettement du discours d'Amina, qui est encore étudiante à Sciences-Po au moment de l'entretien :

Là, à Sciences-Po j’ai du mal... j’ai du mal vraiment. Enfin, je me sens vraiment... En plus j’ai intégré directement en deuxième année, alors que franchement j’aurais préféré refaire une première année. J’ai fait un semestre dans un état pas possible, donc j'en ai rien retiré, même si je l’ai validé.

Pourquoi «dans un état pas possible»?

Parce que j'étais clairement en dépression. C'est ce que je te disais tout à l'heure. Je suis arrivée à Paris. J’ai eu tout... Enfin c'est peut être moi qui m’en fais toute une montagne aussi parce qu'ils ne vont pas me manger à Sciences-Po mais il y a vraiment un truc... J'étais pas bien. Quand j'étais dans ces murs, enfin c'est con parce que voilà ils m'ont rien fait, au contraire ils m'accueillent, ils sont gentils! Mais j'étais pas bien. Et quand je vois les gens, je n'y arrive pas. 
Et en plus quand j'essaye de parler, je vois bien qu'on a pas du tout les mêmes préoccupations. Mais voilà, on n’a pas les mêmes préoccupations du tout. Du tout, du tout. Donc je ne peux pas me lier avec quelqu'un qui... enfin il y a sûrement des gens avec qui je pourrais. Mais je sais pas. Franchement... On n’a pas les mêmes références. Et puis on n’a pas les mêmes préoccupations, donc on ne va pas percevoir les choses de la même manière. Et les choses qui sont importantes pour moi, pour eux ça va être dérisoire. Et vice-versa. Et on ne va pas avoir les mêmes valeurs aussi. Je pense que ça joue vachement. Et puis je suis peut-être d'une nature pas superficielle aussi, c'est clair. Alors à la fois je me dis tu généralises, c'est pas bon, et à la fois... Franchement. Mais donc voilà... Il y a sûrement des gens très intéressants dans le tas, mais bon... (Amina, étudiante à Sciences-Po, $2^{\mathrm{e}}$ année).

Cet extrait d'entretien met bien en évidence la force du choc provoqué par la découverte d'une altérité sociale dont Amina ne soupçonnait pas l'ampleur avant d'en faire l'expérience. Mais son discours révèle surtout qu'elle regarde encore ce «nouveau monde» avec les schèmes de perception de son milieu d'origine. L'opposition entre «eux» et «nous » structure fortement l'ensemble de son discours et vient témoigner d'une volonté forte de résistance à l'acculturation aux schèmes de pensée majoritaires au sein de la grande école dans laquelle elle étudie. Devenir membre du nouveau groupe va donc supposer tout un travail de soi sur soi pour parvenir à rompre les identifications au milieu d'origine et s'identifier progressivement à un autre milieu.

\subsection{TROISIÈME ÉTAPE: QUESTIONS D'APPARTENANCE}

La troisième étape, qui a généralement lieu au cours des études supérieures, consiste en une interrogation sur la place occupée dans l'espace social. Dans un certain nombre de cas, il s'agit d'un moment de «crise» : la fréquentation d'un milieu social différent, combinée à une socialisation anticipatrice à des positions sociales dominantes, provoque un brouillage des appartenances qui peut être fortement déstabilisant. L'étudiant en mobilité sociale doit en effet faire face à un «double calendrier social» (Pasquali, 20ı) dont la gestion est loin d'être évidente. À ce moment de la vie, les liens avec le milieu d'origine, et notamment la famille, restent forts : même si l'étudiant dispose de bourses ou s'autofinance par des «petits boulots», la famille est encore perçue comme le «filet de sécu- 
rité » en cas de coup dur. De plus, le foyer ayant été quitté récemment (quand il a été quitté), les liens affectifs avec les parents et la fratrie demeurent structurants : l'étudiant continue de se définir en grande partie par rapport à ces liens. Pourtant, dans le même temps, la réussite de ses études est partiellement liée à sa capacité à se projeter dans les positions sociales auxquelles ses études sont censées le mener. Cette socialisation par anticipation implique souvent l'entrée dans un processus de déculturation des schèmes d'action et de perception du milieu d'origine qui sont stigmatisés au sein du milieu d’arrivée. Ce processus de déculturation va nécessairement de pair avec un processus d'acculturation aux schèmes dominants. À ce moment plus qu'à aucun autre la tension entre milieu d'origine et milieu d'arrivée est à son comble, et elle est donc plus difficile que jamais à gérer.

Il n'est ainsi pas étonnant que la période des études soit celle qui entraîne le plus souvent la mobilisation de registres tels que ceux de la honte, de la gêne, de la jalousie, de la rancœur, de la souffrance, de la «double absence», de la solitude, etc. Nos entretiens réalisés auprès d’enfants d’ouvriers étudiant à Sciences-Po Paris sont à cet égard extrêmement révélateurs : les narrations de soi sont quasiment toutes marquées par une interrogation sur «la place occupée», débouchant sur des constats explicites ou implicites de «double absence», de non-appartenance à un groupe en particulier, de n'être «ni ici, ni là-bas ». Ainsi, la difficulté qu’a Alexandre à se situer lui-même lorsqu'il parle de sa ville d'origine ou de Paris est assez révélatrice de cette «double absence » :

Dans le domaine politique, ces gens-là,... là-haut,... on se sent un peu à l'écart. Nous quand on dit Paris,... les gens qui sont montés à Paris, qui ont réussi, c'est un peu... c'est comme si nous on avait un peu échoué. Eux...ils ont fait des études, leur avenir est assuré... tandis que là-haut quand tu perds ton travail, quand t'as un travail qu'est pas très bien payé et que tu le perds... ben il faut se débrouiller. Et ça ben je ne sais pas si on en a toujours bien conscience ici de ça, si les élèves qui sont ici en ont conscience. Alors que chez nous... Donc je sais pas... c'est pour ça aussi qu'on est un peu plus méfiants par rapport aux choses. Parce qu'on sait qu'on n'a pas un avenir tout tracé, un avenir sûr. Parce que justement à cause du manque de qualification, à cause du manque d'études... qui ne permettent pas de faire des projets à long terme. Et je ne sais pas si les élèves ils s'en rendent compte. Vraiment. Je ne sais pas. Peut être je me trompe. Je ne sais pas. J'dis pas l'inverse (Alexandre, étudiant à Sciences-Po, $\mathrm{I}^{\mathrm{e}}$ année). 
Il est assez significatif de voir que, dans cet extrait d'entretien, Paris est tantôt décrit du point de vue de ses parents («Nous quand on dit Paris ») et tantôt décrit du point de vue d'un étudiant parisien («ici»). De même sa ville d'origine est parfois décrite d’un point de vue extérieur («là-haut») ou d'un point de vue intérieur au groupe («chez nous»). Cette difficulté à se situer donne lieu, chez Alexandre, à une oscillation entre une volonté d'identification à sa ville d'origine et une nécessité de se reconnaître comme présent «ici», comme étudiant de Sciences-Po. Cela conduit donc à une opposition entre un «ici, eux» et un «là-haut, chez nous», opposition dans laquelle l'étrangeté est proche et la familiarité éloignée.

Cette période de crise peut être éphémère, ne durer que quelques mois, le temps d'un «ajustement». Elle peut même parfois passer inaperçue, notamment quand un certain nombre de facteurs (idéologie familiale, exposition progressive et sans rupture à l'altérité sociale, etc.) contribuent à une transition «douce». Mais, dans d'autres cas, ce moment de crise peut gagner une certaine permanence et durer plusieurs années.

Par ailleurs, il est important de noter que les récits rétrospectifs de nos interviewés sur la période pendant laquelle ils ont douté de leur place dans la société tendent à être fortement reconstruits ou, tout du moins, les difficultés rencontrées à cette époque de leur vie semblent souvent avoir été oubliées. Il est notamment frappant de voir la grande différence entre les discours recueillis auprès des étudiants de Sciences-Po, qui décrivent avec une grande acuité les aléas de leur scolarité à Sciences-Po, et le discours de nos interviewés plus âgés, qui ont beaucoup plus de mal à décrire leur expérience des études supérieures. Les personnes qui se sont engagées dans des stratégies narratives «légitimistes» tendent ainsi à minimiser les souffrances connues pendant cette période. Il n’est pas rare que, lors de certains entretiens, il soit nécessaire d’insister, de «creuser», pour obtenir des témoignages des souffrances vécues, ou des difficultés d’ajustement à cette période.

Adrienne, dont le père était ouvrier-sellier aux usines Citroën et la mère gardienne d'immeuble a eu «la chance» de grandir dans un immeuble bourgeois du quartier des Ternes. Plusieurs des habitants de l'immeuble la recevaient régulièrement chez eux, la conseillaient pour ses choix d'orientation et l'aidaient 
dans son travail. Elle a été scolarisée dans une école et un lycée majoritairement fréquentés par la grande bourgeoisie parisienne. Pendant toute la première partie de l'entretien, elle dit n'avoir jamais vraiment ressenti de différence forte entre elle et les autres étudiants. Au moment où nous l'interrogeons sur ses études à l'ENA, elle nous décrit cette période de sa vie comme relativement heureuse. Jusqu'à ce moment de l'entretien, tout le récit de sa trajectoire semblait donner l'image d'un parcours lisse, sans heurts et sans aucun problème lié à la position sociale de ses parents. Elle semble même un peu surprise par nos questions centrées autour des différences de classe et elle nous déclare: «Ça m’énerve l'image de l'ENA comme un milieu uniquement bourgeois et uniformisateur ! » Le récit de son parcours de réussite est en effet marqué par un refus constant (et qui semble très sincère) d'évoquer le moindre sentiment d'un décalage entre son milieu d'origine et son milieu d'arrivée. Pour elle, son milieu d’origine c'est avant tout ses parents, qui l'ont élevée dans le «culte du travail» et de la réussite scolaire. Son quartier d'origine est un quartier bourgeois et ses amies d'enfance sont essentiellement issues des classes supérieures. Elle n’hésite pas à affirmer : «si moi j’y suis arrivée, les autres le peuvent aussi. Je ne suis pas exceptionnelle. Quand on veut, on peut. » Pourtant, alors que tout laissait croire qu'une idéologie familiale très légitimiste et une enfance dans un environnement immédiat extrêmement bourgeois avaient certainement conduit à une transition extrêmement douce (du fait d'une acculturation précoce aux normes de ce milieu), une question semble subitement lui remémorer des éléments qu’elle confesse avoir oubliés. Selon elle, l'insistance de nos questions lui a permis de se rappeler des éléments d'une période de sa vie qu’elle avait «effacée de sa mémoire »:

Je me rendais compte du décalage entre mon milieu et mon immeuble. La conséquence de cela est que j’ai acheté des tonnes de bouquins de savoir-vivre à cette époque [l'époque du lycée]. Je voulais savoir comment me comporter... J'étais timide et pas à l'aise... J'avais complètement oublié que j'avais eu ce problème (Adrienne, 59 ans, ENA interne, haut fonctionnaire). 
Adrienne sera très lapidaire sur ce point et n'en dira pas plus, peut-être parce que cette anecdote introduit une dissonance dans la cohérence de son récit de vie. Mais ce subit retour à la mémoire d'une période de sa vie qui avait été en quelque sorte refoulée montre bien que, d'une manière consciente ou non, il y a une part de reconstruction des évènements passés dans le récit qui est fait du parcours de réussite. Les éléments qui sont livrés à l'intervieweur sont souvent ceux qui permettent d’assurer au récit toute sa cohérence. Il est ainsi difficile de faire la part des choses entre la sincérité d'une narration mettant l'accent sur une transition heureuse et sans problème, et les stratégies de dissimulation de cette souffrance, stratégies visant parfois à consolider l'idée que l'on est «à sa place » et que l'on n'a rien à se reprocher. Si cette hypothèse d’une dissimulation ne peut être démontrée, elle doit néanmoins être envisagée dans l'analyse qui est faite des narrations de réussite.

Enfin, cette période d'intense oscillation entre deux mondes est souvent marquée par la tentation de dissimuler ses origines. Nos interviewés, bien que très hésitants à confesser une telle chose, sont très nombreux à nous avouer avoir régulièrement caché la profession de leurs parents ou leur quartier d'origine. Les récits de nos interviewés laissent entendre que ce comportement, témoignant d'une certaine «honte» de leur milieu d'origine, est plus fréquent au moment des études supérieures et du début de la carrière et que, selon les trajectoires, il peut se prolonger bien plus tard. Le sociologue Robert Granfield (I99I) a ainsi réalisé un travail ethnographique qui dévoile avec finesse le caractère structurant de ces stratégies de dissimulation chez les étudiants de law school issus de milieu populaire qui, pour la plupart, voient leur origine sociale comme un stigmate.

Si les stratégies d'évitement ou de dissimulation constituent, à ce moment de doute sur les appartenances, un comportement relativement fréquent dans le cadre du nouveau groupe, il faut noter qu'un comportement inverse est également fréquent dans le cadre du groupe d'origine. En effet, un certain nombre de nos interviewés suggèrent qu'ils ont cherché à dissimuler à leur groupe d'origine qu'ils avaient changé, notamment en opérant un changement de code (code switching) en fonction du contexte dans lequel ils se trouvaient. Ce type de comportement consiste à adopter des manières «populaires » lorsqu'on 
est auprès des membres de son groupe d'origine afin de leur cacher que l'on a changé et ne pas passer pour un «bourgeois», un «intello» ou tout simplement quelqu'un qui «les méprise» en les renvoyant à leur position sociale. Le fait qu'à ce moment de l'existence on peut avoir aussi bien «honte» de son milieu d'origine que de son nouveau groupe montre bien l'incertitude de la position dans laquelle se trouvent ces personnes.

\subsection{QUATRIĖME ÉTAPE: L'AJUSTEMENT AU NOUVEAU MILIEU}

Pendant le temps des études supérieures ou au cours des premières années de vie professionnelle, un nouveau cap est généralement franchi. Les personnes en mobilité sociale prennent acte de l'éloignement de leur milieu d'origine. Elles reconnaissent que leur nouveau statut social est profondément différent de celui de leurs parents. Ce constat froid et lucide appelle à la mise en place de stratégies d’ajustement au nouveau statut, afin d'échapper aux problèmes provoqués par le sentiment de double-absence.

Si l'ajustement au nouveau statut est un processus qui se joue dès les premiers moments de la socialisation comme nous avons pu le voir précédemment, c'est néanmoins à partir du moment où le changement de statut est reconnu et acté que la personne va commencer à déployer un discours de justification de la position qu'elle occupe. Tant que l'on a un pied dans chacun des deux mondes et que les études n'ont pas encore été converties en un statut professionnel prestigieux, il est difficile de s'investir dans un travail de stabilisation de son identité. L’enjeu est différent une fois le nouveau statut social confirmé. Constatant l'éloignement de son milieu d'origine, la personne en mobilité doit légitimer cette prise de distance ou, tout du moins, elle doit lui donner sens afin de ne pas en être victime. L'observateur peut alors noter qu'il existe toute une variété de stratégies d'ajustement qui ont en commun de permettre une stabilisation de la façon de penser sa place dans l'espace social. Pour des raisons évidentes de place, nous ne pouvons présenter ici de manière détaillée ces différentes modalités d’ajustement. Il nous semble cependant intéressant de donner à voir au lecteur des exemples de discours de nos interviewés évoquant la sortie de ce moment pendant lequel ils s'interrogeaient sur leur place dans l'espace social. 
Ces discours sont souvent réalisés sur le mode de l'accession à «un stade supérieur de maturité », à un nouveau niveau de conscience.

Pour Yves, la sortie de ses incertitudes sur sa place dans la société est présentée comme liée à la «découverte» des «aspects positifs» de la bourgeoisie. Son discours donne l'impression d'une véritable révélation:

Après on apprend à découvrir une classe sociale qu'on ne connaît pas, qui est celle de la bourgeoisie cultivée. Et, en fait, ce qui est très troublant au début, c'est de s'apercevoir que c'est souvent ces gens là qui sont les plus sympas.

Comment ça?

C'est ce que je vous disais tout à l'heure sur les Pinçon-Charlot. Je dis souvent, et c'est très politiquement incorrect que de dire ça: souvent ce sont des gens qui réussissent, qui sont brillants, à la fois beaux physiquement et extrêmement sympathiques. C'est-à-dire qu'ils ont tout. Les stéréotypes concernant les bourgeois consistent à dire : ils sont hautains, etc. Mais même pas. On ne peut même pas leur reprocher ça. Il y en a certains qui ont vécu sans trop de tiraillements sociaux et familiaux, qui ont une certaine douceur, une certaine ouverture, ils sont éclairés et tout ça. On trouve là des gens extrêmement attentifs, aimables, sincères...

Pour vous ce sont plus des modèles que des contre-modèles?

C'est ça. Exactement. J'arrive à me dire maintenant que, dans les milieux sociaux depuis lesquels je viens, ça peut donner des bons résultats, des gens qui, sans pour autant faire des études, arrivent à avoir de la générosité, etc., etc. Mais, globalement, quand on veut bien manger, on ne mange finalement jamais aussi bien que dans la bourgeoisie. Même sans parler des... Ce sont des gens qui reçoivent bien, qui sont sympathiques. On est bien reçu, on est à l'aise, on mange bien, les gens sont attentionnés, etc. Alors que parfois dans d'autres milieux sociaux, c'est plus dur... Moi quand je vais bouffer chez ma sœur, il n'y a pas... On s'aperçoit après tout que les mœurs de la bourgeoisie, c'est quand même bien. Mais, comme Bourdieu disait que la classe ouvrière reproduit un modèle bourgeois, la bibliothèque, etc. Mais bon... non... Je ne sais pas mes goûts ont changé. Enfin, non: c'est quand même mieux!! (Yves, 3I ans, doctorant en architecture, enseignant-chercheur). 
On voit bien à travers cet extrait d'entretien comment, chez Yves, la sortie de son interrogation sur la place qu'il occupe dans la société est allée de pair avec sa prise de conscience que «les mœurs de la bourgeoisie, c'est quand même bien ». Il lui a fallu accepter cet énoncé pour échapper au sentiment de double-absence et commencer à développer un discours de justification de sa nouvelle position.

La sortie de la période d’incertitude sur la place occupée dans l'espace social est souvent marquée par l'arrêt des stratégies de dissimulation de l'origine sociale qui sont l'un des symptômes les plus évidents d'une «crise des appartenances ». De la même manière, cette période est marquée par une plus grande acceptation de l'image de soi que l'on renvoie à son milieu d'origine. Nos interviewés opèrent ainsi une distinction entre ceux qui les «classent», les «rangent» dans la case des bourgeois et les «vrais amis » ou les membres de la famille qui continuent à voir en eux ce qu’ils ont toujours été. Earl insiste fortement sur la redéfinition qu'il a du opérer de ses liens avec les membres de son milieu d'origine:

Mes liens avec mes amis et ma famille ont dû être redéfinis. Ma relation avec mon meilleur ami... Quand on était jeune, c'était le gars que les filles aiment, le gars avec qui il fallait sortir parce qu'on avait de longues et bonnes conversations. Et après les rôles se sont inversés. Et ils devaient s'inverser. Il voulait rester où il était et maintenant j’ai beaucoup mieux réussi que lui. Donc on a redéfini notre relation... Les gens du quartier me voient toujours comme le petit enfant au nez qui coule qui traîne dans le quartier... Rien n’a changé. Il n'y a pas besoin de leur coller ça sous le nez pour qu'ils le sachent. Si c'étaient vraiment tes amis, ils le comprennent. Et il y a d'autres personnes qui ont du ressentiment. Mais tu t'en fous! Tu ne les fréquentes pas. Et tu redéfinis tes liens... Jusque dans ta propre famille (Earl, 54 ans, MBA, directeur d'une entreprise de haute technologie).

Pour Tom, la sortie des affres de la «crise des appartenances » a nécessité un cheminement différent. Il raconte son enfance et le début de ses études comme un moment pendant lequel il était «un fils loyal mais un peu schizophrène», en raison de son envie de rester attaché à son milieu d'origine tout en ayant le désir d'être ailleurs, loin de sa famille, à vivre de nouvelles expériences. C'est en acceptant que sa traversée de l'espace social faisait de lui quelqu'un de profondé- 
ment unique et en réalisant qu'il lui fallait «forger» une identité complètement inédite qu'il a pu atténuer les effets les plus gênants de cette schize identitaire:

L'une des phrases de James Joyces dans Portrait of the Artist as a Young Man est: «Je veux façonner dans la forge de mon âme la conscience incréée de ma race» [I want to forge in the smithy of my soul the uncreated conscience of my race]. C'est un peu comme si tu ressentais le fait que, d'une manière spirituelle et intellectuelle, tu avais créé en toi une sorte de mentalité qui est très différente, à la fois en termes de psychologie et de mentalité intellectuelle. Et puis tu ressens un sentiment de changement intérieur très fort. Un changement intellectuel et spirituel très fort. Et Joyce décrit ça en termes de ferronnerie. Tu la mets dans le feu. Tu forges une nouvelle conscience. Et c'est vrai! Tu forges une nouvelle conscience. À la fois intellectuellement et spirituellement. Et peut-être même plus. Et tu ressens une tension. Mais ça, tu vis avec... (Tom, 50 ans, doctorat d'anthropologie, lecturer).

Les mots de Tom décrivent avec beaucoup de justesse le défi que doit relever la personne en mobilité sociale: il lui faut faire le deuil de ses appartenances passées et chercher à stabiliser une nouvelle identité. Si Tom affirme avoir développé une «mentalité » complètement inédite, d’autres réinvestissent des modèles préexistants. Mais quelles que soient les modalités d’ajustement au nouveau statut, la personne en mobilité doit se faire «idéologue». En effet, le sentiment d'être à sa place n'allant pas de soi, les personnes en mobilité sociale sont amenées à justifier elles-mêmes leur nouvelle place. Ces personnes doivent être capables de tenir un discours réflexif, quand la plupart des gens n'ont pas à faire l'effort d'expliquer pourquoi ils sont là où ils sont. Une forte mobilité sociale implique nécessairement un discours de soi sur soi et l'incapacité à produire un tel discours condamne à devoir endurer les tourments d'une position incertaine entre deux mondes. Le simple fait de dire que l'on est nulle part, ni ici ni là-bas, et de l'assumer peut suffire : ce qui importe c'est d'être en mesure d’apporter une réponse solide et ferme à la question «Où suis-je? ». 


\subsection{CINQUIÈME ÉTAPE: LA DISPARITION DE LA TENSION}

La dernière des étapes de l'expérience de la mobilité sociale ascendante correspond au moment où le sentiment de tension entre milieu d'origine et milieu d’arrivée disparaît. Le milieu d'origine n'est alors absolument plus considéré comme une référence à partir de laquelle on se définit. Les schèmes d'action et de perception du milieu d'origine ont été parfaitement assimilés, et les traces de la socialisation primaire sont à peine décelables. Le milieu d’arrivée semble alors n'être plus que le seul groupe qui «compte».

Cette étape ne correspond cependant pas à un idéal-type qui ne pourrait être que postulé, mais jamais vérifié. Nous ne faisons pas là l'hypothèse d’un idéal-type de l'acculturation «parfaite » au milieu d’arrivée : une telle situation supposerait en effet que l'histoire passée n'ait plus d'influence et qu'elle ait été substituée par une sorte d’amnésie. Nous décrivons plutôt ici une étape ultime de l'expérience de la mobilité sociale au cours de laquelle la personne est si bien ajustée à son milieu d’arrivée que les forces de rappel du milieu d'origine n'ont plus aucun effet. L'amnésie du milieu d'origine demeure un idéal-type dont l'occurrence est peu probable et, dans les faits, la mémoire du groupe d'origine est donc toujours-là, toujours présente. Mais, dans le cas des personnes qui se situent au niveau de cette «cinquième étape », l'appartenance au groupe d'origine ne constitue plus qu'un simple épisode d'une histoire personnelle qui se raconte à partir de référents bien différents, un épisode qui, tout au mieux, peut-être instrumentalisé avec plus ou moins de cynisme.

Marie-Paule, fille d'une dactylo et d'un cheminot, est aujourd'hui avocate d’affaires dans un cabinet renommé, après avoir connu une brillante carrière dans la haute administration qui l'a amenée à occuper des postes à très hautes responsabilités. Aujourd'hui, elle ne se définit plus par rapport à son milieu d'origine: «J’ai changé de côté. J’appartiens à ce milieu maintenant. » Selon elle, ce changement «s'est fait progressivement»: «Si je retournais aujourd'hui avec des gens de mon milieu d'origine, ils m'identifieraient comme une bourgeoise. Et je le suis. » Pour elle, «être issu de milieu populaire, ça ne compte plus. C'est trop loin. » Aujourd'hui ses parents sont décédés et elle n’a plus aucun contact ni familial, ni amical avec des personnes de milieu populaire. Les personnes avec 
qui elle entretient des relations amicales et sociales sont exclusivement issues des classes supérieures, et même de l'élite parisienne et internationale. Elle prend acte assez sereinement de son changement qu'elle explique ainsi: «Je soupçonne que, insensiblement, sans m’en rendre compte, mes gestes se sont modifiés au contact de ce milieu». Marie-Paule ne se définit plus par rapport à son milieu d'origine et celui-ci n’est plus qu’un thème parmi d’autres de sa mémoire.

Cette cinquième étape n'est pas systématique, et une majorité des personnes interviewées se situeraient plutôt au niveau de la quatrième étape. Elle concerne essentiellement les personnes les plus avancées dans leur carrière. Cette ultime étape suppose une incorporation tellement forte des discours de justification, que ceux-ci ne parviennent plus à être saisis comme tels. Ils apparaissent en effet tellement «naturels» que, parfois, la personne interviewée semble nier le fait que la tension entre son milieu actuel et son milieu d'origine puisse avoir été un enjeu central à un moment de sa vie. De la justification, on est alors passé à une affirmation d'un mode de vie qui va de soi. Cette naturalisation de la justification ne peut se réaliser qu'à certaines conditions: il faut que l'ensemble de l'environnement immédiat de la personne en mobilité sociale (environnement familial, professionnel, amical, urbain) soit assonant et harmonique. Une trop forte dissonance dans l'environnement immédiat et quotidien risque en effet d'entraîner une définition de soi sur le mode de la justification, ce qui en révèlerait alors la dimension construite.

Néanmoins, même chez ceux qui se situent au niveau de cette cinquième étape, la façon de définir sa place dans l'espace social continue à se distinguer de celle de ceux qui sont «nés dans ce milieu». Si les différences sont parfois subtiles, voire même imperceptibles, leurs schèmes d'action et de perception sont le produit d'une socialisation très différente. La différence réside dans le fait que les personnes en très forte mobilité sociale ont dû, à un moment ou à un autre, justifier (à elles-mêmes ou à leur entourage) la position qu'elles occupaient quand les personnes en situation de reproduction sociale, ou qui ont connu une mobilité moins forte, ont plus rarement besoin de faire cet exercice. Un tel impératif de justification peut parfois conduire à acquérir une grande virtuosité dans la maîtrise de ces registres de discours. La capacité à 
contrer toutes sortes d’attaques (notamment celles fondées sur la dénonciation des inégalités de classe ou sur la dénonciation de la domination) peut même conduire certains à être, comme le dit l'expression, «plus royalistes que le Roi». On pourrait rapprocher cette virtuosité, qui s'incarne dans une capacité à justifier le moindre aspect de sa vie, de l'hypothèse de «surconformité » (Lipset et Zetterberg, 1964, Hopkins, 1973), hypothèse selon laquelle les personnes en mobilité sociale tendraient à surenchérir dans la conformité afin d'être mieux intégrées dans leur nouveau groupe. On retrouve cette même idée dans l'évocation par Pierre Bourdieu de «l'adhésion fascinée à l'institution» des «miraculés » (Bourdieu, I989, p. 144).

Quoiqu'il en soit, le fait que nous proposions comme ultime étape de l'expérience de la mobilité sociale la disparition de la tension ne va pas de soi, et pose question. En affirmant que le «clivage du moi» est le principe structurant, et donc en quelque sorte l'horizon indépassable, de l'individu en mobilité sociale ascendante ${ }^{10}$, Bernard Lahire (1998, p. 46-52) prend ainsi une position apparemment directement contradictoire avec la nôtre. Son affirmation est cependant difficile à confirmer ou à infirmer. Difficile à infirmer car si l'on part du principe, relativement consensuel, et que nous avons exposé plus haut, selon lequel l'amnésie du milieu d'origine est impossible (à l'exception peut-être de quelques cas cliniques), alors il demeurera toujours une influence du milieu d'origine qui sera plus ou moins fortement refoulée, influence qui est donc la cause d'un certain «clivage du moi». En effet, le clivage entre deux répertoires de schèmes d'action et de perception est redoublé d'une relation de domination qui rend impossible leur fusion et qui condamnera toujours les dispositions acquises dans le nouveau milieu à entrer en contradiction avec les dispositions,

I0 Bernard Lahire a certainement raison, dans le cadre de sa réflexion, d'affirmer que les personnes en forte mobilité sociale ne constituent pas le meilleur cas à partir duquel bâtir une théorie de la pluralité des dispositions. Mais, l'analyse qu'il réalise de l'expérience de la mobilité sociale dans le but d'appuyer son raisonnement est quelque peu réductrice. À trop insister sur l'importance du clivage du moi, il laisse en effet entendre qu'il est difficile pour les personnes en mobilité de faire coexister pacifiquement différents répertoires de schèmes d'action. Son analyse tend ainsi à dessiner un effet de la mobilité sociale (le «clivage du moi»), là où nous soutenons qu'il existe une pluralité d'effets de la mobilité sociale. 
jamais complètement disparues, du milieu d'origine ${ }^{11}$. Cependant, réduire l'expérience de la mobilité sociale à l'expérience d'un «clivage du moi», c'est s'interdire de penser la complexité de l'expérience de la mobilité. La permanence des schèmes d'action et de perception issus du milieu d'origine peut être plus ou moins forte et il est important de rendre compte de ces variations de degrés.

\section{REMARQUES CONCLUSIVES}

L’intérêt d'une approche de l'expérience de la mobilité sociale en termes de «grandes étapes» est avant tout de renouveler le regard sur le débat autour des effets «anomiques» de la mobilité sociale. En effet, cela permet de saisir à quel point la mobilité sociale ne produit pas des effets qui perdurent de manière homogène dans le temps. La période autour des études supérieures condense ainsi toutes les contradictions auxquelles doivent faire face ces personnes. La prise en compte de la fluctuation dans le temps de l'expérience de la réussite doit ainsi amener à un renouvellement partiel des paradigmes structurant la littérature sur l'expérience de la mobilité : en fonction de l'âge des personnes étudiées, les effets que feront ressortir les travaux peuvent varier considérablement. Si l'hypothèse d'anomie et l'hypothèse d'acculturation sont souvent présentées comme «alternatives», l'une ou bien l'autre sortant vainqueur de l'analyse sociologique, une approche diachronique de l'expérience de la mobilité devrait permettre de les considérer comme potentiellement «cumulatives» (voir aussi Naudet, 20I I).

Par ailleurs, une approche en termes de «grandes étapes» contribue à un renouvellement du regard porté sur le phénomène de reproduction sociale. Dans les études sur l'expérience de la mobilité sociale, l'interrogation la plus classique consiste à prendre comme point de départ le groupe d'origine afin d'évaluer à quel point les personnes en ascension demeurent marquées par leurs dispositions d'origine et continuent, malgré leur changement de statut

II Précisons cependant que Bernard Lahire prend bien soin de nuancer son propos en affirmant que «ce conflit intérieur peut être apaisé et ne conduit pas toujours (cela dépendra de l'histoire propre à chaque acteur) aux plus grandes dissociations psychiques et aux plus extrêmes tortures mentales » (1998, p. 49). II n'en dit cependant pas plus sur la façon dont ce conflit peut être apaisé. 
social, à être irrémédiablement marquées du sceau du populaire. Notre approche, moins immédiatement évidente, consiste plutôt à se demander comment ces personnes parviennent, malgré tout, à incorporer les dispositions des classes dominantes. En effet, la reproduction sociale est également liée à la capacité qu'ont les classes dominantes à demeurer stables malgré l'arrivée de nouveaux membres en leur sein. Si les personnes originaires des milieux populaires demeuraient vraiment fidèles à leur groupe d'origine, il serait beaucoup trop «dangereux» pour l'élite en place de les accueillir en leur sein. Pour que l'ordre social soit préservé, il est donc important que les personnes originaires des classes populaires se «convertissent» et incorporent autant que possible les dispositions caractéristiques des membres déjà en place.

Notre typologie des grandes étapes de la mobilité entend ainsi participer à la compréhension du processus par lequel une personne issue de milieu dominé parvient à devenir dominante. Elle s'inscrit en cela dans la continuité de la métaphore, développée par Joseph Schumpeter, de la classe sociale comme un autobus qui, à la fin de son trajet, ne contiendrait plus les mêmes voyageurs quau départ (1972, p. I83). La mobilité sociale ascendante affecte certes la composition des classes supérieures, mais ne suffit pas à les liquider. Au contraire, l'entrée dans la classe dominante implique nécessairement un long processus d’apprentissage des dispositions légitimes au sein de ce groupe, processus garant de la pérennité des frontières de classe.

\section{BIBLIOGRAPHIE}

ASHFORD Sheena, 1989, «Social Mobility. A Test of the Marginality and Acculturation Hypotheses», International Journal of Sociology and Social Policy, 8-6, p. 25-47.

BLAU Peter M., 1956, «Social Mobility and Interpersonal Relations», American Sociological Review, 21-3, p. 290-295.

-, DUNCAN Otis Dudley, 1967, The American Occupational Structure, New York, The Free Press. 
BOURDIEU Pierre, 1974, «Avenir de classe et causalité du probable», Revue Française de Sociologie, 15-I, p. 3-42.

-, 1989, La Noblesse d'État: Grandes Écoles et esprit de corps, Paris, Éditions de minuit.

ELLIS Robert A., LANE W. Clayton, 1967, «Social Mobility and Social Isolation: A Test of Sorokin's Dissociative Hypothesis », American Sociological Review, 32-2, p. 237-253.

FREUD Sigmund, 1973, «Le roman familial des névrosés». in Jean Laplanche (dir.), Névrose, psychose et perversion, Paris, PUF, p. 157.

GAULEJAC Vincent (de), 1987, La Névrose de classe: trajectoire sociale et conflits d'identité, Paris, Hommes et Groupes Éditeurs.

GRANFIELD Robert, 1991, «Making it by faking it: Working-Class Students in an Elite Academic Environment», Journal of Contemporary Ethnography, 20-3, p.331-35I.

HOGGART Richard, 1970, La Culture du pauvre, Paris, Éditions de Minuit.

HOPPER Earl, 198I, Social mobility: a study of social control and insatiability, Oxford, Blackwell.

LAHIRE Bernard, 1995, Tableaux de familles, Paris, Gallimard.

-, 1998, L'Homme pluriel: les ressorts de l'action, Paris, Nathan.

LAREAU Annette, 2000, Home advantage: Social class and parental intervention in elementary education, Lanham, Rowman \& Littlefield.

-, 2003, Unequal childhoods: Class, race, and family life, University of California Press.

LUBRANO Alfred, 2004, Limbo: Blue-Collar Roots, White-Collar Dreams, Hoboken, Wiley and Sons.

LUCKMANN Thomas, BERGER Peter, 1964, «Social Mobility and Personal Identity», European Journal of Sociology, 5-2, 1964, p. 331-344.

MERCKLÉ Pierre, 2005, «Une sociologie des "irrégularités sociales" est-elle possible?», Idées, 142, p. 22-29.

NAFF Katherine C., 1998, «Progress toward Achieving a Representative Federal Bureaucracy: The Impact of Supervisors and Their Beliefs», Public Personnel Management, 27, p. I35-I50. 
NAUDET Jules, 2010, Analyse comparée de l'expérience de la mobilité sociale ascendante intergénérationnelle aux États-Unis, en France et en Inde, Thèse de doctorat sous la direction de Marco Oberti, soutenue à l'IEP Paris, le 2 décembre 2010.

-, 201I, «L'expérience de la mobilité sociale: plaidoyer pour une approche par le discours», Bulletin de Méthodologie Sociologique, II2-1, p. 43-62.

-, 2012. «Mobilité sociale et explications de la réussite en France, aux États-Unis et en Inde», Sociologie, 3-I, p. 39-59.

NEWMAN Katherine S., 1989, Falling from Grace: The Experience of Downward Mobility in the American Middle Class, New York, Vintage Books.

PAGE Paul, 1994, «African-Americans in Executive Branch Agencies», Review of Public Personnel Administration, 14-I, p. 24-5I.

PASQUALI Paul, 2010, «Les déplacés de I"'ouverture sociale": Sociologie d'une expérimentation scolaire», Actes de la recherche en sciences sociales, 183-3, 2010, p.86-105.

PASSERON Jean-Claude, 1993, «Portrait de Richard Hoggart en sociologue», Enquête. Cahiers du CERCOM, 8, p. 79-III.

PEUGNY Camille, 2009, Le Déclassement, Paris, Grasset.

POLIAK Claude, 1992, La Vocation d'autodidactes, Paris, L'Harmattan.

SCHUMPETER Joseph, 1972, Impérialisme et classes sociales, Paris, Éditions de Minuit.

SCHÜTZ Alfred, 2003, L'Étranger, Paris, Allia.

SOROKIN Pitirim, 1927, Social Mobility, New York, Harper and Brothers.

TERRAIL Jean-Pierre, 1990, Destins ouvriers. La fin d'une classe?, Paris, PUF.

TURNER Frederick C. (ed.), 1992, Social mobility and political attitudes: comparative perspectives, New Brunswick, N.J., Transaction Publishers.

ZWEIGENHAFT Richard L., DOMHOFF G. William, 2003, Blacks in the white elite: will the progress continue?, Lanham, Md., Rowman \& Littlefield. 
